\title{
Dynamic Evacuation Routes for Personnel on a Naval Ship
}

\author{
Francisco Pérez-Villalonga ${ }^{1}$, Javier Salmerón ${ }^{2}$ and R. Kevin Wood $^{2}$ \\ ${ }^{1}$ Navy of Spain, Ministerio de Defensa, Arturo Soria 287, 28033, Madrid, Spain \\ ${ }^{2}$ Naval Postgraduate School, Monterey, CA, 93943, U.S.A.
}

\begin{abstract}
We propose a "dynamic escape route system" for emergency evacuation of a naval ship. The system employs signals that adapt to the causative contingency and the crew's physical distribution about the ship. A mixed-integer nonlinear programming model, with underlying network structure, optimizes the evacuation process. The network's nodes represent compartments, closures (e.g., doors, hatches) and intersections, while arcs represent various types of passageways. The objective function integrates two potentially conflicting factors: average evacuation time, and the watertight and airtight integrity of the ship after evacuation. A heuristic solves the model approximately using a sequence of mixed-integer linear approximating problems. Using data for a Spanish frigate, with standard "static routes" specified by the ship's designers, computational tests show that the dynamic system can reduce average evacuation times nearly $23 \%$, and can improve a combined measure of ship integrity by up to $50 \%$. In addition, plausible design changes to the frigate yield further, substantial improvements.
\end{abstract}

Keywords: evacuation time; ship integrity; multi-commodity flow; nonlinear network model

\section{INTRODUCTION}

The abandonment of a naval ship is an improbable event, but if it does occur, the ship is likely to be sinking or on fire. Because of this danger, the crew should evacuate the ship's interior as quickly as possible. While evacuating, however, the crew should attempt to maintain the ship's watertight and airtight integrity by leaving open as few doors, hatches and other closures as possible. This paper shows how a system of configurable signals could direct crewmembers to escape routes that would reduce average evacuation time and improve the postevacuation watertight and airtight integrity of the ship, compared to currently used static escape routes.

Static escape routes are those routes implied by signs painted or mounted on a ship's passageways (e.g., corridors, stairways) to guide evacuees to mustering stations (i.e., gathering points), prior to leaving the ship; this system of routes is "information non-adaptive”. A dynamic escape route system (DERS) would also guide evacuees to mustering stations, but would incorporate electronic or electro-mechanical signals that could be configured to account for specific damage conditions, e.g., blocked passageways, and for the crew’s physical distribution 
DRAFT, 1 July 2008

about the ship; such a system is "information adaptive.” By coupling extra information with appropriate signaling, the dynamic system has a clear potential for reducing evacuation times and thereby improving the crew's safety, compared to the static routes.

The International Maritime Organization (IMO) establishes security procedures for merchant and passenger ships to comply with mandatory Safety of Life at Sea (SOLAS) regulations [14]. The North Atlantic Treaty Organization (NATO) has recently developed analogous regulations as part of its Naval Ship Code (NATO Naval Armaments Group [18]; see Chapter VII). Although these regulations are not mandatory for NATO's member nations, at least one member, the United Kingdom, has adopted them [15]. Excerpts from the Code state:

Naval vessels shall be adequately designed, constructed, equipped, maintained and provided with procedures for the Escape, Evacuation and Rescue of all embarked persons following all foreseeable emergency situations and damage conditions ([18], p. 85).

Escape and Evacuation Analysis and Escape and Evacuation Demonstration shall ensure that effectiveness of escape and evacuation measures are optimised ([18], p. 88).

Clearly, the static escape route system that naval ships currently use cannot be reconciled with these regulations.

We do not assume that DERS would optimize escape routes at the time of the contingency (although future research may show this to be possible). Rather, we expect that alternative route configurations would be established in advance. Then, given (a) a specific contingency, (b) the ship's damage condition or expected damage condition, and (c) the crew's physical distribution about the ship, the ship's damage-control officer would select the evacuation plan that best fits the situation. Alternatively, an automated system could make this selection with the damagecontrol officer's oversight. The signals would probably consist of illuminated (possibly electromechanical) arrow signs indicating directions of travel, and would be activated according to the pre-stored configuration that best suits the contingency. 
DRAFT, 1 July 2008

We note that several dynamic escape-route systems are already commercially available for fire-protection in structures such as hospitals, airports and office buildings. For instance, the Dynamic Escape Routing System (INOTEC Sicherheitstechnik GmbH [12]) uses visual signals, while EVACom (Schneider Intercom [24]) uses auditory signals. To our knowledge, these systems respond to information from sensors such as smoke detectors, but do not account for the physical distribution of a structure's occupants. However, researchers who study the evacuation of these structures certainly realize that better escape routes could be developed by taking this distribution into account (Pu and Zlatanova [21]).

DERS is especially appropriate for a naval ship, because (a) the crew of such a ship is a welltrained and exceedingly familiar with its ship, and therefore should have no difficulties following special signals, (b) unlike passengers on a passenger vessel, the location of crewmembers on a naval ship is known quite accurately in most situations, and (c) the ship’s physical condition, i.e., “damage condition,” resulting from an accident or hostile action, should be better defined than on a commercial vessel, because a naval ship is built with extensive damage control in mind.

The DERS we propose will signal an escape route based on the solution of a mathematicalprogramming model that minimizes a weighted function of average evacuation time and a "shipintegrity index," for a given damage condition and crew distribution. (For simplicity, we use “integrity” hereafter to mean "watertight and airtight integrity.”) The ship’s post-evacuation integrity is important because (a) the longer a damaged ship stays afloat, the better chance its crew has to be rescued, and (b) if a ship maintains its integrity, it may not sink at all and can be salvaged and returned to service, at a cost well below that of a constructing new ship.

A weighted-sum objective function has the benefit that its optimal solutions are "efficient" (i.e., “nondominated,” or “Pareto-optimal”) for its objectives (e.g., Ehrgott [6], pp. 24, 68). Solving the model for different weights does not guarantee the generation of all efficient solutions; the goal-programming technique of constraining one objective and optimizing the other can help find additional efficient solutions (e.g., Ehrgott [6], pp. 98-110). But, our computational 
DRAFT, 1 July 2008

experience shows that the weighted-sum approach produces efficient solutions that leave only modest gaps in the trade-off curve ("efficient frontier") for the two objective functions. Thus, when it comes time to establish an appropriate tradeoff, the decision-maker can choose from a wide range of options which we, as analysts, will have provided.

A potential objection to the weighted-sum approach is that it mixes goals measured in different units (e.g., Rosenthal [23]). In our case, we mix units of ship integrity with units of time. The former units may be viewed as having a partly economic value-try to keep the ship afloat so it can be salvaged - while the latter correlates primarily with saving human lives. However, military planners make such tradeoffs all the time, at least implicitly, and our approach can provide such planners with a wide range of solutions from which to choose an appropriate tradeoff. If computational results on another ship type should exhibit large jumps in one objective as weights change only modestly, then a goal-programming approach could be considered.

The remainder of this paper is organized as follows. Section 2 describes the ship-evacuation process and reviews relevant literature. Section 3 explains our modeling assumptions and presents the mathematical formulation of the problem. This section also describes the heuristic algorithm we use to solve the problem approximately. Section 4 presents computational results for the DERS model applied to a Spanish frigate, under several contingency scenarios. Section 5 summarizes the paper and provides conclusions.

\section{SHIP EVACUATION AND EVACUATION MODELS}

\subsection{Situational Awareness and Damage Information}

The command structure of a naval ship will normally have a good understanding of the ship's status, including where its personnel are located and the physical condition of the ship. Three concepts are key to understanding this:

- The watch condition of readiness, or simply "watch condition," ordered by the commanding officer (CO), determines which stations are manned and to what extent, 
DRAFT, 1 July 2008

based on the organizational bill (“watch, quarter and station bill”) of the ship. That is, the watch condition defines, to a greater or lesser degree, the physical distribution of the crew about the ship. Representative instances are "General Quarters,” which applies during combat and under emergencies, and "Peace-time Cruising.” Under General Quarters, with few exceptions, the location of every crewmember will be known precisely, because they will be at their pre-assigned battle stations. During Peace-time Cruising, only the location of crewmembers standing watch will be known precisely, but good estimates should available for the number of crew in sleeping areas and in the mess.

- The material condition of readiness, or simply "material condition," also ordered by the $\mathrm{CO}$, defines the degree of access to various areas of the ship. All closures are marked with a letter, which determines whether the closure is open or closed for the ordered material condition (United States Government Printing Office [27]). For instance, material condition ZEBRA applies during combat operations and provides the greatest degree of integrity but the least accessibility across the ship: closures marked "X," "Y," and "Z” are all closed in this case. On the other hand, material condition XRAY applies when the ship is in no apparent danger: only closures marked with " $\mathrm{X}$ " are closed. YOKE is an intermediate condition, used in wartime but non-combat situations, and requires "X" and "Y" closures to be closed. In effect then, material condition determines a ship’s pre-contingency level of integrity.

- Should a ship receive any damage, her damage-control officer orders repair parties to assess and report on damage, and to try to extinguish fires or block flooding. The goal is to maintain the ship’s fighting capability and to enable an eventual return to port. Except in the case of certain catastrophic damage, the damage-control officer, and thus the CO, should have an accurate estimate of the damage to the ship, including (a) which passages 
DRAFT, 1 July 2008

are blocked or partially blocked by damage or by repair-party work, and (b) the condition of mustering stations.

Analyzing the sequence of events that may lead to ship abandonment, while taking the three concepts above into account, we identify two general cases in which pre-programmed evacuation plans would apply:

- Case 1, delayed abandonment. If struck by a missile or mine, or if some other contingency arises, the ship will probably not be abandoned immediately. The crew would first be ordered to General Quarters (Hays [11]). Then, the damage-control officer repair parties and other crew would assesses the damage, and begin damage control, if possible. At this stage, dynamic escape routes could be based on (a) the location and extent of the damage reported by the repair parties and compiled by the damage-control officer, (b) an accurate knowledge of the crew's physical distribution, known insofar as most crewmembers would be at their general-quarters stations (exceptions would depend on the location of damage-control parties, casualties, and the fact that some stations may be inaccessible because of damage or fire), and (c) the known status of most closures. (General Quarters implies material condition ZEBRA, although damage and damagecontrol efforts might modify the status of some closures.) In this situation, escape routes would direct crewmembers from their known locations, along undamaged passageways to undamaged mustering stations, and could limit evacuation along routes needed by repair parties.

- Case 2, immediate abandonment. A ship can be damaged so severely by torpedoes, missiles or other weapons that its loss becomes apparent within a few minutes, or even seconds, of incurring the damage. In this situation, DERS would incorporate the best data possible for the applicable watch and material conditions. DERS might also be able to incorporate some information about actual damage (for example, from visual observation 
DRAFT, 1 July 2008

from the bridge) or anticipated damage (for example, a ship that is threatened by mines can at least anticipate that any damage will occur below the waterline.)

Case 1 would apply in the majority of instances in which a ship must eventually be abandoned, and we see that this "standard case" would also present the most accurate data for selecting a near-optimal DERS evacuation plan.

Case 2 would be the "fallback case,” which would be used if the ship receives an impact with little or no warning. For instance, during the Falklands War of 1982, the Argentine cruiser $A R A$ General Belgrano sank only minutes after being struck by torpedoes from a British submarine (Woodward [28], pp. 149-163). The General Belgrano detected neither the submarine nor its torpedoes before the first torpedo exploded. Even in this case, however, DERS would be exploiting partial information, specifically, (a) a complete or partial picture of crew distribution, depending on the watch condition, and (b) the state of each closure not affected by the impact, depending on the material condition. For instance, the General Belgrano was in watch condition Wartime Cruising when it was struck (Armada Argentina [1]), and thus, its crew's physical distribution should have been known in some detail.

DERS will likely yield better escape routes than a static system, even with incomplete information as in Case 2. With the addition of sensors that identify impassable or damaged passageways, and that determine the exact number of crewmembers in each compartment, the system could respond equally well in all situations.

\subsection{Models for Ship Evacuation}

The evacuation process for occupants of any structure, e.g., building, aircraft, passenger ship, offshore platform, and even for inhabitants of a geographical region, has been modeled in the literature using two basic approaches:

1. An optimization model searches for optimal routes for evacuees, who are typically treated as a single, homogeneous commodity in which individual behavior can be ignored. These 
DRAFT, 1 July 2008

models are typically linear or nonlinear programs that may be viewed as network-flow models with side constraints (e.g., Chalmet et al. [2], Chiu et al. [3] and Choi et al. [4]).

2. A discrete-event simulation takes individual movement and uncertain behavior into account, trying to represent realistically the decisions made during the evacuation process (e.g., Fire Safety Engineering Group [7], Taaffe et al. [26]).

We choose optimization because the trained crew of a naval ship can be expected to move where directed, i.e., as controlled "flows" of individuals, and because optimization is better at providing the prescriptive, escape-route solutions we desire. However, our optimization model, even in a simplified, linearized form, is substantially more complicated than the models in [2]-[4]. In particular, evacuees are not homogeneous because crewmembers in a given compartment at the time of evacuation must follow a single evacuation route as a group. This means that our model requires multi-commodity-flow constructs, not just single-commodity constructs. Furthermore, the modeling of groups requires the use of binary flow variables which, along with a knapsack capacity constraint for each mustering station, imply that the linearized model is no easier to solve than the "multiple knapsack problem.” The multiple knapsack problem is NP-hard (Karp [16]), and thus it appears that our model is, in fact, a difficult-to-solve, nonlinear, integerprogramming model.

The next section develops an optimization model of the ship-evacuation process that (a) accounts for the ship's watertight and airtight integrity, (b) adapts data regarding the movement speeds of evacuees to a homogeneous population, (c) explicitly incorporates the effects of impediments such as sealed closures, blocked passageways and counter-movements ("counterflows”) of repair parties, and (d) identifies an evacuation route for each group of crewmembers. 
DRAFT, 1 July 2008

\section{MATHEMATICAL MODEL AND ALGORITHM}

\subsection{Overview and Preliminaries}

We represent a ship’s “enclosure” by a directed network $H=(N, A)$, derived from technical and construction drawings: this is the "DERS network." Nodes $N$ represent compartments, closures and intersections of passageways, and $\operatorname{arcs} A$ represent passageways along with directions of travel that evacuees could conceivably use. Source nodes represent compartments where crewmembers are located when “Abandon Ship!” is ordered, and “muster nodes” represent mustering stations from where the crewmembers will abandon the ship.

The occupants of each compartment, i.e., a group, define a unit of supply of a single commodity, in a multi-commodity flow model defined on $H$. Each muster node connects, via an artificial arc, to a super-sink with one unit of demand for each commodity. This represents the reasonable assumption that all crewmembers in a group will follow the same route and reach the same mustering station (although the model will choose which mustering station). Joint capacity constraints on these mustering-station arcs-each commodity applies a conversion factor from "group" to "number of crewmembers in the group"-represent upper and lower bounds on the number of crewmembers that should gather there. Upper bounds reflect the limited capacity of lifeboats located at the mustering stations, and possibly the limited number of lifejackets there; lower bounds reflect the minimum number of crewmembers needed to manage lifeboats properly. (In high conditions of readiness, each crewmember may preemptively don an inflatable lifejacket. In this case, the supply of lifejackets at each mustering station would become irrelevant, although the capacity of lifeboats would continue to constrain any evacuation.)

The model seeks to minimize an objective function that incorporates two goals represented by an evacuation index $T$ and a ship-integrity index $I$. The index $T$ reflects the average evacuation time for all crewmembers, while the index I reflects the number of closures that are opened to aid evacuation. These two goals conflict with each other to some extent: opening (“unsealing”) a 
DRAFT, 1 July 2008

watertight or airtight door may speed evacuation, but it may also degrade the ship's integrity. Once opened, the closure may not be closed ("resealed”) for reasons discussed below, and an open closure reduces integrity because it increases the volume of the ship that is subject to flooding or fire damage.

The model is a nonlinear, mixed-integer program with an underlying network structure. The model is difficult to solve exactly for two key reasons:

- The speed $s_{g i j}$ at which evacuee group $g$ traverses arc $(i, j)$ depends on the total number of evacuees traversing the arc at roughly the same time. We adopt the speed function recommended by the IMO for passenger ships, but without age or gender corrections (International Maritime Organization [13]; see also Figure 1). This function depends on

o type of arc, e.g., corridor, companionway, trunk (a "companionway" is a staircase between decks, and a "trunk" is a vertical passageway fitted with a ladder, sometimes specifically intended to aid escape),

o the direction (horizontal, up, or down), and

o the concurrent flow of evacuees traversing an arc (the number of people moving past a given point per unit time per unit of unimpeded passage width).

Because flow speed depends on flow quantity, the continuous relaxation of the model is nonlinear and non-convex. We note that many other approaches exist to represent speed and congestion effects, especially in the vehicle-traffic literature; see Hall [10] who provides an overview of standard models. More recently, several researchers (e.g., Nishi and Takahashi [19] for a vehicle-traffic model, Zhang et al. [29] for a work-flow model) have suggested the use of staircase functions. These might prove computationally useful given our approach of mixed-integer programming, but we have not pursued that topic. 
DRAFT, 1 July 2008

- Opening a watertight hatch or other closure on a naval ship is not as simple as opening a door in a building, and can take many seconds. Thus, the first group to reach a sealed closure at node $i$ incurs a delay $t_{i}$, corresponding to time required to open the closure. Subsequent groups do not incur that delay directly, because we assume that the closure remains open. (Standard naval doctrine actually decrees that sailors who pass through a closure should reseal that closure after themselves. However, in an emergency situation, this doctrine may not be followed consistently, or damage or flooding may make resealing impossible. We must make some assumption, and we choose this one because it is conservative with respect to ship integrity.) To model such delays explicitly requires constructs much like those suggested to model certain job-shop scheduling problems; unfortunately, these models are notoriously difficult to solve (Shi and Pan [25]).

\subsection{Optimization Model, DER-NL}

The following mixed-integer nonlinear program defines our Dynamic Escape-Route model (DER-NL) for identifying optimal evacuation routes under given watch and material conditions. We will solve it approximately using the heuristic algorithm described subsequently.

\section{Indices}

$i, j \in N$

$g \in G$

$i_{g}^{0}$

$N^{M} \subset N$

$N^{D} \subset N$

$(i, j) \in A$ nodes, including an artificial super-sink denoted $i^{+}$

groups, which consist of crewmembers originally located at the same node (compartment)

origin node for group $g, i_{g}^{0} \in N$

nodes representing mustering stations

nodes that require opening a closure for the first group that traverses the node (depends on the material condition of the ship)

directed arcs in $H=(N, A)$; $A$ includes artificial arcs $a=\left(i, i^{+}\right)$for $i \in N^{M}$ 
DRAFT, 1 July 2008

C

set of indices for counter-flow constraints, defined as:

$$
C=\left\{\left(g, g^{\prime}, i, j\right) \mid g, g^{\prime} \in G ; g \neq g^{\prime} ; i<j ;(i, j),(j, i) \in A\right\}
$$

(Note: The heuristic procedure only builds a small subset of $C$ "on the fly," but the final subset guarantees that all counter-flow constraints are satisfied.)

\section{Parameters [units]}

$c_{i} \quad$ capacity of muster node $i \in N^{M}$ [number of evacuees]

$v_{g} \quad$ size of group $g$ for the given watch condition [number of evacuees]

$d_{i j} \quad$ length of arc $(i, j)$ [meters] (Note: When $i$ is an origin node, $d_{i j}$ includes a correction factor based on the average difficulty for a crewmember in the compartment represented by the node $i$ to reach the appropriate exit. Also, note that $d_{i i^{+}} \equiv 0 \forall i \in N^{M}$.)

$S_{i j}(F)$ speed on arc $(i, j)$ as a function of the total concurrent flow $F$ on the arc [meters/second] (See Figure 1.)

$\omega_{i} \quad 1$ if closure $i$ is open in the current material condition, and 0 otherwise

$\hat{t}_{i} \quad$ time required to open the closure at node $i \in N^{D}$ [seconds] (this is 0 if closure $i$ is open in the specified material condition)

$\alpha \quad$ objective-function weight for the trade-off between evacuation time and ship integrity

$\bar{t} \quad$ reference value used in the objective function for normalizing the evacuation-time index [seconds] (We use $\bar{t}=180$ seconds based on the standard duration of the emergency breathing devices.)

\section{Derived Data}

$\gamma_{i} \quad$ non-negative weight for closure at node $i \in N^{D}$, which reflects the closure's contribution to the ship’s watertight or airtight integrity. For simplicity, we classify each closure as contributing to watertight integrity exclusively, or to airtight integrity exclusively, or to 
DRAFT, 1 July 2008

neither. Then, $\gamma_{i}$ is computed as $\beta \rho_{i}^{\text {water }} / n^{\text {water }}$ for watertight closures $i$, and as $(1-\beta) \rho_{i}^{\text {air }} / n^{\text {air }}$ for airtight closures, where

o $\rho_{i}^{\text {water }}$ is the fractional amount that closure $i$ contributes to the ship's watertight integrity $\left(\rho_{i}^{\text {water }}=1.0,0.8\right.$ and 0.2 for watertight doors, hatches and scuttles, respectively),

o $\quad \rho_{i}^{\text {air }}$ is defined similarly for airtight integrity (with additional data, $\rho_{i}^{\text {water }}$ and $\rho_{i}^{\text {air }}$ could reflect more detail, such as the volume of the compartment or passageway that a closure affects),

$0 n^{\text {water }}$ and $n^{\text {air }}$ are the total number of watertight and airtight closures, respectively, for the given material condition, and

o $\quad \beta$ reflects the relative importance of watertight versus airtight integrity. ( $\beta=0.75$ for all tests. This is somewhat arbitrary, but reflects the naval emphasis on watertight integrity, which assures that a ship stays afloat.)

\section{Decision variables [units]}

$f_{g i j} \quad 1$ if group $g$ traverses arc $(i, j)$, and 0 otherwise

$S_{g i j}$ speed of group $g$ while traversing arc $(i, j)$ [meters/second] (Note: $s_{g i i^{+}} \equiv \mu \forall g \in G, i \in N^{M}$ for some large constant $\mu>0$.)

$t_{g i j} \quad$ time when group $g$ starts traversing arc $(i, j)$, if it does, and 0 otherwise [seconds]

$y_{g i} \quad 1$ if group $g$ is the first group crossing a closed closure $i \in N^{D}$, and 0 otherwise

$T \quad$ evacuation-time index (used for notational clarity)

I Ship-integrity index (used for notational clarity)

$z^{*} \quad$ optimal objective-function value (used for notational clarity) 
$\mathbf{f}, \mathbf{s}, \mathbf{t}, \mathbf{y}$ vector forms of $f_{g i j}, s_{g i j}, t_{g i j}$ and $y_{g i}$, respectively.

\section{Formulation (DER-NL)}

(0) $z^{*}=\min \alpha T+(1-\alpha) I$,

where $T \equiv \frac{1}{\bar{t}}\left(\left(\sum_{g} v_{g}\right)^{-1} \sum_{\substack{(i, j) \in A \mid \\ j \neq i^{+}}} \sum_{g} v_{g} \frac{d_{i j}}{S_{g i j}} f_{g i j}+\sum_{i \in N^{D}} \sum_{g} t_{i} y_{g i}\right)$ and $I \equiv \sum_{i \in N^{D}} \sum_{g} \gamma_{i} y_{g i}$, subject to

$$
\begin{aligned}
& \sum_{j \mid(i, j) \in A} f_{g i j}-\sum_{j \mid(j, i) \in A} f_{g j i}=\left\{\begin{array}{rl}
1, & \text { if } i=i_{g}^{0} \\
-1, & \text { if } i=i^{+} \\
0, & \text { if } i \in N \backslash\left\{i_{g}^{0}, i^{+}\right\}
\end{array}, \forall g \in G\right. \\
& \sum_{g \in G} v_{g} f_{g i i^{+}} \leq c_{i}, \quad \forall i \in N^{M} \\
& f_{g i j}+f_{g^{\prime} j i} \leq 1, \quad \forall\left(g, g^{\prime}, i, j\right) \in C \\
& y_{g i} \in\{0,1\} \forall g, i ; \quad f_{g i j} \in\{0,1\} \forall g, i, j \\
& (\mathbf{f}, \mathbf{s}, \mathbf{t}, \mathbf{y}) \in X,
\end{aligned}
$$

and where $X$ implicitly defines the following relationships among $\mathbf{f}, \mathbf{s}, \mathbf{t}$, and $\mathbf{y}$ :

For each $i \in N^{D}$ and $g \in G$

$$
y_{g i}= \begin{cases}1 & \text { if } g=\underset{g^{\prime} \in G}{\operatorname{argmin}}\left\{t_{g^{\prime} i j} \mid f_{g^{\prime} i j}=1,(i, j) \in A\right\} \\ 0 & \text { otherwise. }\end{cases}
$$

Let $\left(i_{k}, j_{k}\right), k=1,2, \ldots K_{g}$, be the ordered sequence of consecutive arcs traversed by group $g \in G$. Then:

$$
t_{g i_{k} j_{k}}= \begin{cases}0, & \text { if } k=1 \\ t_{g i_{k-1} j_{k-1}}+\frac{d_{i_{k} j_{k}}}{S_{g i_{k} j_{k}}}, & \text { if } i_{k} \notin N^{D}, k=2, \ldots, K_{g} \\ t_{g i_{k-1} k_{k-1}}+\frac{d_{i_{K} j_{K}}}{S_{g i_{k} j_{k}}}+\hat{t}_{i_{k}} y_{g i_{k}}, & \text { if } i_{k} \in N^{D}, k=2, \ldots, K_{g}\end{cases}
$$

For each $(i, j) \in A$ and $g \in G$ such that $f_{g i j}=1$, let $G_{g i j}=\left\{g^{\prime} \in G \mid\right.$ groups $g$ and $g^{\prime}$ traverse $(i, j)$ concurrently $\}$.

To determine $G_{g i j}$, we first compare times $t_{g i j}$ and $t_{g j p}$ at which a given group $g$ should enter and leave an arc $(i, j)$ given current speed estimates, where $p$ is the next node after $j$ in group $g$ 's path. Groups $g$ and $g^{\prime}$ are then deemed to 
DRAFT, 1 July 2008

traverse $(i, j)$ concurrently if both groups enter or exit the arc nearly simultaneously, or one group appears to overtake and pass the other: $\left(\left|t_{g^{\prime} i j}-t_{g_{i j}}\right| \leq \varepsilon\right) \vee\left(\left|t_{g^{\prime} j p}-t_{g j p}\right| \leq \varepsilon\right) \vee\left(t_{g^{i j}} \leq t_{g^{\prime} i j} \wedge t_{g j p} \geq t_{g^{\prime} j p}\right)$ $\vee\left(t_{g^{\prime} i j} \leq t_{g i j} \wedge t_{g^{\prime} j p} \geq t_{g j p}\right)$, where $\varepsilon$ equals 5 seconds in all tests.

Let $F_{g i j}=\sum_{g^{\prime} \in G_{g i j}} v_{g^{\prime}}$ be the total flow (number of evacuees) concurrent with group $g$ to traverse arc $(i, j)$. Then, $s_{g^{\prime} i j}=S_{i j}\left(F_{g i j}\right) \forall g^{\prime} \in G_{g i j}$. (See Figure 1.)

\section{Description of DER-NL}

DER-NL seeks to minimize the objective function (0) based on evacuation-time index $T$ and ship-integrity index $I$. Index $T$ represents the average evacuation time for all groups, normalized by the reference time $\bar{t}$, while index $I$ represents a weighted and normalized count of the number of closures that are opened by the evacuees.

Constraints (1) represent flow-balance constraints for all groups and nodes. These constraints ensure that all the evacuees leave their respective origin nodes (sources) and reach some mustering station, if this is feasible.

Constraints (2) limit the total number of evacuees allowed to assemble at each mustering station, based on the availability and capacity of the lifeboats and lifejackets at each station.

Constraints (3) prohibit counter-flows, i.e., two groups moving in opposite directions on any arc. Counter-flows are unacceptable because escape signs should only point in one direction: once configured, escape signs must remain fixed during the evacuation process, and cannot vary based on which group is traversing the arc. These constraints do not prevent two groups that arrive at a node from departing in different directions, however. Allowing such movement may be reasonable for groups that arrive from different directions, since they would see different signs; but this reflects a model limitation, otherwise. (None of the routes we identify in computational 
DRAFT, 1 July 2008

tests exhibits such problematic movements, but if needed, constraints and binary variables could be added to the model to force a unique forward direction of travel at each node.)

Constraints (4) ensure that arc flows and closure openings are binary decisions.

Equation (5.a) determines whether or not a group is the first group to traverse a node with a sealed closure. Equation (5.b) calculates the time at which a given group starts traversing a given arc. Equation (5.c) calculates the speed at which a given group traverses an arc, based on the approximate number of evacuees that share that arc during each time interval.

Part of the requirements enforced by equations (5) could be avoided by using a "time-phased network” as proposed by Ford and Fulkerson [9]. This network would notably increase our model's size, yet it would not completely eliminate the nonlinearity associated with concurrent flows on arcs. The next section proposes a heuristic algorithm to deal with this and related issues.

\subsection{Heuristic Algorithm for Solving DER-NL}

We solve DER-NL approximately using an iterative heuristic algorithm that incorporates ideas similar to successive linear programming (e.g., Fletcher and Sainz [8]). In each iteration $\ell$, a mixed-integer linear program (MIP), DER-L ${ }^{\ell}$, approximates DER-NL. DER-L ${ }^{\ell}$ replaces nonlinear terms in DER-NL, such as $1 / s_{g i j}$, by fixed values derived by post-processing the solution to the previous approximating model, DER-L ${ }^{\ell-1}$. (Of course, values for the first approximating model, DER-L ${ }^{0}$, are also specified in the heuristic.) For efficiency, DER-L ${ }^{\ell}$ only includes those counter-flow constraints required to avoid inconsistencies in previous solutions. The iterative process repeats until the approximating model requires no additional adjustments. Unlike standard sequential linear programming, we do not add penalty terms nor do we enforce trust regions: experience indicates that the process converges to a feasible solution in a few iterations without such embellishments. We define the procedure formally below. Note that the heuristic yields objective value $\hat{z}$, rather than $z^{*}$. 
DRAFT, 1 July 2008

\section{DER Heuristic Algorithm (DER-HA)}

Step 0 (Initialization): Set

$$
\hat{y}_{g i}:=0, \forall g \in G, i \in N^{D} ; \hat{s}_{g i j}:=S_{i j}\left(v_{g}\right), \forall g \in G,(i, j) \in A ; C:=\varnothing ; \ell:=0 ;
$$

Step 1 (Optimization): Solve the current approximating model:

$\left(\mathrm{DER}-\mathrm{L}^{\ell}\right) \quad \min \quad \mathrm{z}=\alpha T+(1-\alpha) I$

$$
\begin{array}{ll}
\text { s.t. } & (1)-(4) \\
& (7)-(9)
\end{array}
$$

where (3) is only applied to elements in $C$, and (6)-(8) are defined as follows:

(6) $s_{g i j}=\hat{s}_{g i j}, \forall g \in G,(i, j) \in A$ (implemented via substitution),

(7) $\sum_{g^{\prime} \in G} y_{g^{\prime} i} \geq \sum_{j \mid(i, j) \in A} f_{g i j}, \forall g \in G, i \in N^{D}$

(8) $y_{g i} \geq \sum_{j \mid(i, j) \in A} f_{g i j}, \forall g \in G, i \in N^{D} \mid \hat{y}_{g i}=1$.

Assume DER-L $\mathrm{L}^{\ell}$ yields solution vector $(\hat{\mathbf{f}}, \hat{\mathbf{s}}, \hat{\mathbf{y}}, \hat{z})$.

If $\ell=0$, then let $\underline{z}:=\hat{z}$;

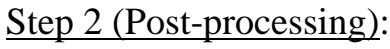

2.a: For all $(i, j) \in A$ and $g \in G$, estimate all $\hat{t}_{g i j}$ using (5.b) and update $\hat{s}_{g i j}$ and $\hat{y}_{g l}$ with values consistent with (5.a)-(5.c). (See details below.)

2.b: Update counter-flow set for the next iteration:

$$
C:=C \cup\left\{\left(g, g^{\prime}, i, j\right) \mid \hat{f}_{g i j} \cdot \hat{f}_{g^{\prime} j i}=1\right\} ;
$$

\section{Step 3 (Convergence):}

If no update to any $\hat{s}_{g i j}$ or $\hat{y}_{g, i}$, or to $C$, was made in Step 2, then

Print (“The heuristic solution is", $\hat{\mathbf{f}}, \hat{\mathbf{s}}, \hat{\mathbf{t}}, \hat{\mathbf{y}}$ ).

Print (“Corresponding objective value is”, $\hat{z}$, “and lower bound is”, $\underline{z}$ ).

STOP.

Else, set $\ell:=\ell+1$ and return to Step 1 .

Constraints (6) fix all speeds in DER-L ${ }^{\ell}$ to those estimated from the previous iteration, except for the first iteration, i.e., when $\ell=0$. Constraints (7) force some group that passes through a nominally sealed closure to be charged with the time penalty for opening it. After the 
DRAFT, 1 July 2008

first iteration, constraints (8) require the first group to reach a closure in the previous iteration to be charged with the penalty if that group persists in using the closure in the incumbent iteration.

For the first iteration, the speed of any group $g$ traversing arc $(i, j)$ is set to the maximum possible, $s_{i j}=S_{i j}\left(v_{g}\right)$. This assumes no other group traverses that arc concurrently with group $g$. Constraints (8) do not apply in this first iteration, and constraints (7) clearly imply a relaxation of the "real constraint" that the first group reaching a sealed closure be charged with the time penalty for opening it. Given the ideal traversal speeds and the relaxed constraints, the optimal objective value from iteration $\ell=0$ provides a lower bound on $z^{*}$.

Iterations continue until (a) no significant variation exists between the values of $\hat{\mathbf{s}}$ and $\hat{\mathbf{y}}$ produced by DER-L $\mathrm{L}^{\ell}$ and their corresponding post-processed values, and (b) no counter-flows are observed. "No significant variation” in condition (a) means that the algorithm has identified a stable set of routes and travel speeds, and that the group charged with opening any closure is, in fact, the first group to arrive at that closure.

The data updates in the post-processing step must be described in more detail to understand how the algorithm functions. Using the evacuation routes identified in the solution to DER-L we must estimate the time at which each group traverses the nodes and arcs in its individual evacuation route to provide values for the next iteration. We estimate these times through a iterative sequence of group-speed computations that are based concurrent-flow estimates, while also accounting for the times required to open sealed closures. Specifically, at the end of Step 1, the algorithm will have returned have returned $(\hat{\mathbf{f}}, \hat{\mathbf{s}}, \hat{\mathbf{y}}, \hat{z})$ as the solution to DER-L $\mathrm{L}^{\ell}$, and the postprocessing step, Step 2.a, estimates $\hat{t}_{g i j}$ and updates $\hat{s}_{g i j}$ and $\hat{y}_{g l}$ for iteration $\ell+1$ as follows:

\section{Details for Step 2.a in DER-HA}

2.a.1: For each group $g$, start at origin node $i_{g}^{0}$, and use the incumbent evacuee flow $\hat{\mathbf{f}}$ to find the ordered sequence of arcs $\left(i_{k}, j_{k}\right), k=1,2, \ldots K_{g}$, traversed by group $g$. 
DRAFT, 1 July 2008

2.a.2: Let $\mathbf{s}:=\hat{\mathbf{s}}$ and $\mathbf{y}:=\hat{\mathbf{y}}$.

2.a.3: For each group $g$, and for $k=1,2, \ldots K_{g}$, compute the arc-traversal time $t_{g i_{k} j_{k}}$ using equation (5.b).

2.a.4: For each $i \in N^{D}$ and $g \in G$, use the new values for $t_{g i j}$ to update $\mathbf{y}$ as indicated in equation (5.a).

2.a.5: For each arc $(i, j)$ traversed by group $g$, compute concurrent flow $F_{g i j}$ and update $\mathbf{s}$, all as in equation (5.c).

2.a.6: If any update to $\mathbf{y}$ or $\mathbf{s}$ has taken place in Step (2.a.4) or (2.a.5), return to Step (2.a.3).

2.a.6: Let $\hat{\mathbf{s}}:=\mathbf{s}$ and $\hat{\mathbf{y}}:=\mathbf{y}$, and use $\hat{\mathbf{s}}, \hat{\mathbf{y}}$ and $\hat{\mathbf{f}}$ to update $\hat{z}$ as indicated in the objective function (0).

2.a.7: Continue with Step 2.b in DER-HA (to update counter-flows).

In computational tests, we find Steps 2.a.3 through 2.a.6 terminate after at most five iterations, and thus Step 2.a contributes only negligibly to the overall run time of DER-HA.

\section{COMPUTATIONAL RESULTS}

This section applies DER-NL, and the heuristic solution algorithm DER-HA, to a number of evacuation scenarios for a 5,800 tonne Spanish frigate, the F-101 Álvaro de Bazán. We have implemented DER-HA using the XPress-MP 2006 optimization suite [5] (optimizer version 17.01.02), on a Dell Latitude D410 computer running at $2 \mathrm{GHz}$ with 2 Gb of RAM.

The longest run for any scenario takes no more than one hour and 20 major iterations. The average model solves in under 10 minutes and in about six iterations of DER-HA. At least 95\% of computational time is spent solving the MIPs in Step 1 of the algorithm. Because of these run times, DERS would clearly need to be implemented with pre-programmed evacuation plans.

The relative optimality gap, $100 \% \times(\hat{z}-\underline{z}) / \underline{z}$, ranges from $0.1 \%$ to $2.4 \%$ for the "standard scenarios," where we use $\alpha=0.75$. Thus, we can claim near-optimality of the heuristic solutions for these standard scenarios. The gap may increase, however, for small values of $\alpha$ that 
DRAFT, 1 July 2008

emphasize ship integrity and which therefore lead to substantial congestions along certain escape routes and correspondingly weak lower bounds.. For example, when $\alpha=0.01$, some combinations of material and watch conditions lead to provable optimality gaps of nearly $20 \%$.

Overall, we note that DERS does achieve some of its improvements by reducing congestion. To demonstrate this, let us define a "(directed) traversal” as one group traversing a particular arc in its evacuation route, and define a traversal as "congested" if the traversal is made at less than maximum speed. We find that (a) DERS reduces the total number of congested traversals over the static solution from 1,295 to 913 in the Wartime-Cruising (intact-ship) scenario described in Section 4.5, and (b) DERS reduces the number of congested traversals from 425 to 334 in the General-Quarters (USS Stark) scenario described in Section 4.6. DERS also reduces the total number of traversals, congested or not.

The following subsections describe the study scenarios, and compare detailed results for DERS and static routes in those scenarios.

\subsection{Case Study Details}

The Álvaro de Bazán (Figure 2) is the first of four medium-sized multi-purpose frigates ordered by the Spanish Navy. The Navantia (formerly Izar) shipyard built the frigate, which was commissioned in 2002; see Pérez-Villalonga [20] for additional background.

The DERS network for the Álvaro de Bazán comprises 639 nodes and 1,435 arcs. Nodes represent compartments, closures, intersections and mustering stations, distributed on seven decks, with three decks above the main deck and three below. Arcs represent companionways, corridors, trunks and other passageways.

The distribution of the ship's 245 crewmembers across compartments depends on the watch condition ordered by the CO. Table 1 shows significant differences in crew distribution by deck for some representative conditions. For example, the In-port watch condition has most of the crew 
DRAFT, 1 July 2008

resting in cabins and berthing. In this case, the ship's material condition is typically "XRAY," and ship integrity is most relaxed, i.e., many closures are open.

\subsection{Scenario Overview}

We demonstrate the benefits of DERS using a number of scenarios, each defined by watch, material, and damage conditions. Three standard combinations of watch and material conditions are considered: General Quarters with ZEBRA (GQ-Z), Wartime Cruising with YOKE (WC-Y), and In-port with XRAY (IP-X). Each of these combinations differ in both crew distribution (defined by the watch condition) and which closures are initially open (defined by the material condition), so we assess add two hypothetical combinations for initial testing: General Quarters with YOKE (GQ-Y) and Wartime Cruising with ZEBRA (WC-Z). This allows us to explore the separate effects watch and material conditions. Specific damage conditions considered are "intact ship,” "partially blocked passages” and "fully blocked passages.” An intact ship exhibits no substantial damage and has all passageways clear: a fire in the engine room of a ship might require the ship's evacuation yet no passageways (or nearly none) need by blocked or made impassable by a fire. For "partially blocked passages," speed is reduced on certain arcs to represent passageways filled with smoke or partially filled with water, or to represent passages being used by damage-control parties. "Fully blocked passages" represents a situation in which certain passageways are destroyed by fire or explosion, for instance, after the impact of a missile. Arcs representing such passageways are explicitly removed from the DERS network. We assume damage conditions for the Álvaro de Bazán similar to those experienced by the USS Stark in 1987 (Levinson and Randy [17], pp. 16-18) and the USS Cole in 2000 (Raman [22]).

A last test case explores a plausible design change which could improve evacuation times for a given level of ship integrity.

In all scenarios, lifeboat availability determines the maximum physical capacities at the mustering stations. Unless otherwise specified, these capacities are as follows: 147 crewmembers 
DRAFT, 1 July 2008

at the forecastle station, 147 at the flight-deck station, and 36 at the boat-deck station. (Note that the total capacity of 330 lifeboat seats exceeds the ships standard complement of 245 crewmembers by $35 \%$.)

\subsection{Evaluating Static Routes}

We evaluate static routes by solving DER-NL with $f_{g i j}$ fixed to 0 for any arc $(i, j)$ if technical drawings for the frigate contraindicate evacuation in that arc's direction. In no scenario does damage block a passage required by a static escape route. That is, we have not modeled a scenario in which some crewmembers follow the static route defined by technical drawings, find a passage blocked, are forced to find some other escape route, and thereby incur a substantial delay in their evacuation. Analysis of such situations might show even greater benefits for DERS than those reported below.

\subsection{Initial Testing}

Before investigating how DERS can improve ship evacuations over a static escape route system, we want to show how DERS' solutions produce sensible tradeoffs between average evacuation time and ship integrity, depending on how the parameter $\alpha$ is set. To do this, we analyze four intact-ship scenarios that cover GQ-Z, WC-Y, GQ-Y and WC-Z, across a wide range of values for $\alpha$. (Recall that GQ-Y and WC-Y are artificial conditions, used only for this analysis.)

Figure 3 shows the "approximate efficient frontier" for the ship-integrity and evacuation time indices that is generated for each scenario by solving DERS for various values of $\alpha$ between 0.001 and 0.999; “approximate” applies here because DERS is solved with a heuristic. We make the following observations on this figure.

- The curves have the right shape, that is, they are generally convex and decreasing. (The curves would be perfectly convex if we were solving a linear program rather than a nonlinear MIP.) 
DRAFT, 1 July 2008

- The WC-Z curve lies to the left of the WC-Y curve and, likewise for GQ-Z versus GQY. This means that the 12 "Z"-labeled closures, which are open in material condition YOKE but closed under ZEBRA, do not shorten average evacuation times appreciably by being open. (Furthermore, if for some $\alpha$ those 12 closures formed a subset of the optimal set of opened closures, we would see a Z- and corresponding Y-curve cross. This would happen because the condition YOKE would not incur the "fixed charge" for opening those closures.)

- The conclusion above is strengthened by observing this fact: the fastest evacuation times for material condition YOKE lead to noticeably worse integrity indices compared to the fastest times for ZEBRA. (For $\alpha=0.999$, compare GQ-Y to GQ-Z, and compare WC-Y to WC-Z.)

- The substantial differences between the GQ-Y and WQ-Y curves, and between the GQ-Z and WQ-Z curves, indicate that the crew's physical distribution about the ship does influence evacuations substantially. (In fact, the crew occupies more compartments under General Quarters than under Wartime Cruising.)

- If ship integrity is of little concern ( $\alpha=0.999$ ), the ship can be evacuated in roughly the same amount of time, independent of the watch and material conditions considered here.

For simplicity in the remainder of our computational tests, we consider only GQ-Z, WC-Y and IP-X scenarios, and keep $\alpha$ fixed to 0.75 . This allows us to focus on how ship damage affects results, and lets us demonstrate how DERS could be used to help improve a ship's design.

\subsection{Results for Intact-Ship Scenarios}

Table 2 displays overall results for the GQ-Z, WC-Y and IP-X scenarios. Dynamic routes improve the objective-function value by between $16 \%$ and $24 \%$, as a result of improvements in both average evacuation time and ship integrity. Average evacuation time reduces by up to $23 \%$ with DERS, while the integrity index reduces by up to $45 \%$; the individual watertight and airtight 
DRAFT, 1 July 2008

integrity indices reduce by up to $50 \%$ and $11 \%$, respectively. In the GQ-Z scenario the airtight index actually degrades by $11 \%$, but this is offset by a $50 \%$ improvement in the watertight index and a $13 \%$ reduction in evacuation time, for an overall objective-function improvement of $22 \%$, and an overall integrity index improvement of $37 \%$.

Dynamic routes achieve improvements over static routes by changing the direction of select escape-route signs, using fewer so that routes are more direct, and opening fewer closures. For instance, in the GQ-Z scenario,

- DERS reverses sign directions in 13 passageways, which results in 8 passageways being used in opposite directions between the two routing solutions (the static solution does not use 5 of these 13);

- $\quad$ DERS uses 222 total arcs versus 247 for the static solution;

- The two solutions have only 148 "traversed passageways" in common;

- DERS opens 86 total closures versus 105 for the static solution; and

- The two solutions have only 62 opened closures in common.

So, it appears that the relatively modest number of direction-reversals on escape-route signs leads to a dramatically different escape-route solution.

The total number of evacuees that reach each mustering station are similar in the IP-X scenario, for both static and dynamic routes. However, in the GQ-Z and WC-Y scenarios, dynamic routes increase the use of the flight-deck mustering station by $25 \%$ and $6 \%$, respectively. Dynamic routes also use escape trunks for more crewmembers than do static routes. This seems to be a desirable result, as escape trunks offer a fast and protected egress from spaces located in lower decks, without significant watertight degradation.

\subsection{Results for Damaged-ship Scenarios}

"Damaged-ship scenarios" are characterized by a damaged area and the extent to which passageways and closures in that area are disabled. We are interested, in part, in discouraging 
DRAFT, 1 July 2008

flows of evacuees through the passageways used by repair parties, as well as counter-flows between these groups. To do this, we simply decrease $S_{i j}(F)$ along arcs $(i, j)$ used by repair parties. (So, we do not prohibit counter-flows between repair parties and evacuees.) We analyze two examples of damage to the case-study frigate. The damage scenarios are based on two attacks on the U.S. frigates, USS Stark and USS Cole. Table 3 displays results.

\section{Case 1: USS Stark}

In this scenario, the Álvaro de Bazán receives an impact similar to that received by the USS Stark in 1987. The following conditions hold.

- Watch condition: General Quarters or Wartime Cruising. (The USS Stark was at sea at the time of the attack.)

- Material condition: ZEBRA with General Quarters and YOKE with Wartime Cruising.

- Damage condition: Passageways and closures located on Decks 1, 2 and 01, on the bow port side, become impassable. Additionally, speed is significantly reduced for any crewmembers traversing passageways that would be used by repair crews.

Compared to static escape routes, dynamic routes improve the ship-integrity index by $36 \%$ for both scenarios, and reduce the average evacuation time by $8 \%$ and $22 \%$, respectively. DERS does not directly minimize the time that the last evacuee reaches a mustering station, but we note that, in the Wartime-Cruising scenario, this statistic is $32 \%$ smaller for the DERS solution compared to its static-route counterpart; it is $10 \%$ larger in the General-Quarters scenario, however.

\section{Case 2: USS Cole}

In this scenario, the Álvaro de Bazán is moored, port side to pier, and an explosion occurs amidships, close to the waterline. This affects the engine room and other areas. This scenario represents a situation similar to that experienced by the USS Cole in 2000, although the Cole was anchored in a port at the time of the attack, not moored at a pier. Two important facts characterize 
DRAFT, 1 July 2008

this case: most of the crew is resting on the lower decks, and only one ladder, on the flight deck, leads to the pier. The only watch condition considered is In-port; the material condition is XRAY. (Because of potential threats, the USS Cole was actually in a modified ZEBRA condition when it was attacked in the Yemeni port of Aden. We assume that the Álvaro de Bazán is moored in a port that has been deemed safe.)

Under these assumptions, we expect the majority of the crew to evacuate the ship via the flight-deck ladder. To model this situation, we modify the capacity of the forecastle mustering station to $10 \%$ of the total crew (instead of the $60 \%$ assumed in other scenarios), and increase the capacity of the flight deck to $100 \%$. This forces most of the crew to head toward the flight deck from their berthing areas situated on Decks 2 and 3. We assume that lifeboats cannot be lowered, and therefore set the capacity of the boat deck to zero. Repair parties will try to control flooding by pumping water through the escape trunk on the port side of the damaged engine room.

DERS improves the objective-function value by $20 \%$ over the static plan, reduces the average evacuation time by 26 seconds (25\%) and keeps more watertight doors closed. (The last evacuee escapes faster, too.) Dynamic routes take advantage of known usable passageways to avoid congestion, despite the need to route most of the crew to the flight-deck mustering station.

\subsection{Design-Change Scenarios}

Naval architects must demonstrate the effectiveness of a ship's evacuation system during the early steps of the design process, when important changes have less impact on the production budget. DER-NL could be used to evaluate various design alternatives with respect to this effectiveness. To demonstrate this, we consider a design modification to the Álvaro de Bazán that increases the widths of all passageways and closures by $10 \%$, which would enable faster movement by the crew in the case of an evacuation. This modification is always possible, in theory, although it would require that the ship's dimensions be increased or that compartment sizes be reduced. 
DRAFT, 1 July 2008

We explore the new design for an intact ship, and compare results to those presented earlier for DERS, obtained without the design modifications (Table 2). Table 4 presents the comparison. In all cases, the optimal objective-function value improves, especially in the Wartime-Cruising scenario (by 26\%) and the In-port scenario (by 39\%).

\section{CONCLUSIONS}

We have demonstrated the improvements that a dynamic escape route system (DERS) on a naval ship can have over the current paradigm of static routes. DERS plans escape routes that minimize a weighted sum of an evacuation-time index and a ship-integrity index. The first index represents a normalized function of average evacuation time, and the second reflects the number of closures (doors, hatches, etc.) that are opened to facilitate evacuation.

For the Spanish frigate Álvaro de Bazán, average evacuation time across three baseline intact-ship scenarios is reduced by up to $23 \%$, while the ship's integrity is improved by up to 50\%. In some damaged-ship scenarios, these savings are even greater.

We model the evacuation process in DERS using a nonlinear, integer-programming model, “DER-NL.” We solve this model heuristically with provably good results: for standard test cases that balance evacuation speed and ship integrity fairly evenly, the heuristic yields solutions that lie within $3 \%$ of a specially computed lower bound. (When the objective function heavily favors ship integrity, however, this "provable optimality gap” increases substantially, at least in part because the lower bound weakens.)

DERS improves upon a static system because it can account for (a) the physical distribution of the crew (this varies depending on the ship's watch condition, but is typically well defined), (b) obstructed passageways or closures, and (c) the known status of each closure, open or closed. We also demonstrate how DER-NL could help evaluate how design changes to a ship would affect the crew's evacuation speed and the ship's post-evacuation integrity. 
DRAFT, 1 July 2008

Additional study is certainly warranted. In particular, simulation experiments should be carried out to verify the accuracy of the approximations used in DER-NL and to make possible adjustments. It seems unlikely, at least for the frigate used in our computational example, that constraining one objective and optimizing the other would yield substantially different results. However, this goal-programming technique might be useful when analyzing other ship types, and that option should be kept in mind.

Simulation might show that DERS does not improve the evacuation process as much as predicted, at least in some scenarios. But, greater benefits than seen in this paper might accrue in others. For instance, we have not compared DERS to static routes in scenarios in which some static routes are blocked. In such a situation, static routes could result in much backtracking, confusion, congestion and delay, while DERS could lead crewmembers to a direct, quick and safe egress. Finally, we also note that DER-NL could be used during the ship's design process to determine a distribution of life boats at the ship’s mustering stations that best accommodates a range of scenarios.

\section{ACKNOWLEDGEMENTS}

The authors thank CAPT Jeffrey Kline USN (Ret.) for valuable guidance in writing this paper, and thank the Office of Naval Research for its support. We also thank two anonymous referees and the associate editor for their helpful comments.

\section{REFERENCES}

[1] Armada Argentina, Historia del Crucero ARA “General Belgrano” en la Armada Argentina, (2007) http://www.ara.mil.ar/malv_accbelicas_belgrano.asp (Accessed October 2007).

[2] L.G. Chalmet, R.L. Francis, and P. B. Saunders, Network models for building evacuation, Mgmt Sci 28 (1982) 86-105.

[3] Y. Chiu, H. Zheng, J. Villalobos, and B. Gautam, Modeling no-notice mass evacuation using a dynamic traffic flow optimization model, IIE Transactions 39, (2007) 83-94. 
DRAFT, 1 July 2008

[4] W. Choi, H. W. Hamacher, and S. Tufecki, Modeling of building evacuation problems by network flows with side constraints, Eur. J. Oper. Res. 35 (1988) 98-110.

[5] Dash Optimization. XPress MP - Release 2006. (2006) www.dashoptimization.com (Accessed November 2006).

[6] M. Ehrgott, Multicriteria optimization. Springer, Berlin, 2005.

[7] Fire Safety Engineering Group, Exodus: The evacuation modeling tool for the safety industry. University of Greenwich (2006). http://fseg.gre.ac.uk/ (Accessed Nov. 2006)

[8] R. Fletcher, and E. Sainz de la Maza, Nonlinear programming and nonsmooth optimization by successive linear programming. Math Prog 43 (1989), 235-256.

[9] L. R. Ford, and D. R. Fulkerson, Constructing maximal dynamic flows from static flows. Oper Res 6 (1958), 419-433.

[10] F. L. Hall, Traffic stream characteristics, Traffic Flow Theory, N. H. Gartner, C. J. Messer, and K. K. Rathi (Editors), US Federal Highway Administration, Washington, DC, 1996. pp. 2-1 to 2-34.

[11] M. K. Hays, Optimal manning experiment changes damage control philosophy, Fathom, 1215.

[12] INOTEC Sicherheitstechnik GmbH, Dynamic escape routing system (2007). http://www.hormen.cz/Filemanager/File/pdf/inotec_DER_system.pdf (Accessed Oct. 2007)

[13] International Maritime Organization. Interim guidelines for evacuation analyses for new and existing passenger ships (MSC/Circ 1033). (2002).

[14] International Maritime Organization. International Convention for the Safety of Life at Sea (SOLAS), 1974. http://www.imo.org/home.asp?topic_id=424 (Accessed February 2008)

[15] JSP430 (Joint Service Publication), MOD Ship Safety Management Part 3, Naval Authority Regulations, Issue 4. Ministry of Defence, United Kingdom, September 2007. http://www.nakmo.co.uk/OpenDocument.aspx?Type=Library\&ID=606 (Accessed February 2008).

[16] R. M. Karp, Reducibility among combinatorial problems, Complexity of computer computations, R. E. Miller and J. W. Thatcher, (Editors), Plenum Press, New York, 1972, pp. 85-103.

[17] J. Levinson, and L. E. Randy, Missile inbound, Naval Institute Press, Annapolis, 1997.

[18] NATO Naval Armaments Group, Allied Naval Engineering Publication, ANEP - 77, Naval Ship Code (Accessed February 2008) http://www.nakmo.co.uk/OpenDocument.aspx?Type=Library\&ID=556

[19] T. Nishi and D. Takahashi, 8-figured hysteresis loop of OV model, Proc. Traffic and Granular Flow ’01, 2001, pp. 235-242. 
DRAFT, 1 July 2008

[20] F. Pérez-Villalonga, Dynamic escape routes for naval ships. Masters Thesis, Operations Research Department, Naval Postgraduate School (2005).

[21] S. Pu and S. Zlatanova, Evacuation route calculation of inner buildings. Geo-information for disaster management, P. van Oosterom, S. Zlatanova and E. M. Fendel (Editors),. Springer Verlag, Heidelberg, 2005, pp. 1143-1161.

[22] B. Raman, Attack on USS Cole: Background, South Asia Analysis Group, Paper no. 152. http://www.saag.org/papers2/paper152.html (Accessed Feb. 2006)

[23] R. E. Rosenthal, Principles of multiobjective optimization, Decision Sciences 16 (1985) 133-152.

[24] Schneider Intercom, EVACom by Schneider Intercom, (2007). http://www.schneiderintercom.de/go/evacom (Accessed Oct. 2007)

[25] L. Shi, and Y. Pan, An efficient search method for job-shop scheduling problems. IEEE Transactions on Automation Science and Engineering 2 (2005) 73 -77.

[26] K. Taaffe, M. Johnson, and D. Steinmann, Improving hospital evacuation planning using simulation, Proceedings of the 2006 winter simulation conference, Monterey, California, 2006, pp. 509-515.

[27] United States Government Printing Office. Nomenclature of decks. Navy Department, Bureau of Ships (May 1936).

[28] S. Woodward, One hundred days, Naval Institute Press, Annapolis, Maryland, 1992.

[29] M. Zhang, R., Batta, and R. Nagi, Modeling of workflow congestion and optimizing of flow routing in a facility, Working Paper, Department of Industrial and Systems Engineering, SUNY at Buffalo, Buffalo, New York, 2007. 
DRAFT, 1 July 2008

Table 1. Crew distribution for the Álvaro de Bazán, by deck, for the watch conditions of General Quarters, Wartime Cruising and In-port. Values in the table are numbers of crewmembers.

\begin{tabular}{|c|c|c|c|c|c|c|c|}
\hline & 4 & 3 & 2 & $\begin{array}{c}\text { Deck } \\
\text { (Main) } 1\end{array}$ & 01 & 02 & 03 \\
\hline General Quarters & 20 & 5 & 101 & 34 & 59 & 20 & 6 \\
\hline Wartime Cruising & 9 & 59 & 110 & 23 & 32 & 9 & 3 \\
\hline In-port & 0 & 167 & 62 & 14 & 0 & 2 & 0 \\
\hline
\end{tabular}

Table 2. Computational results for intact-ship scenarios. Note that $\hat{z}=\alpha \hat{T}-(1-\alpha) \hat{I}$, where $\hat{z}$ denotes the best value objective value calculated through DER-NL. $\hat{T}$ is the average evacuation time divided by the reference time $\bar{t} ; \hat{I}$ is the ship-integrity index, calculated as a weighed average of the watertight and airtight integrity indices (weights $\beta$ and $1-\beta$, respectively). Test cases use $\alpha=0.75, \beta=0.75$ and $\bar{t}=180$ seconds, unless otherwise specified.

\begin{tabular}{l|rrrrrr}
$\begin{array}{l}\text { Watch condition, material } \\
\text { condition }\end{array}$ & \multicolumn{3}{c}{$\begin{array}{c}\text { General Quarters, } \\
\text { ZEBRA }\end{array}$} & \multicolumn{2}{c}{$\begin{array}{c}\text { Wartime Cruising, } \\
\text { YOKE }\end{array}$} & \multicolumn{2}{c}{$\begin{array}{c}\text { In-port, } \\
\text { XRAY }\end{array}$} \\
& Static & Dynamic & Static & Dynamic & Static & Dynamic \\
\hline Model & 0.40 & 0.31 & 0.41 & 0.31 & 0.49 & 0.41 \\
Objective value $\hat{z}$ & 0.36 & 0.31 & 0.39 & 0.33 & 0.40 & 0.31 \\
Evacuation-time index $\hat{T}$ & 0.52 & 0.33 & 0.47 & 0.26 & 0.73 & 0.72 \\
Ship-integrity index $\hat{I}$ & 64 & 56 & 70 & 59 & 73 & 56 \\
Avg. evac. time (sec.) & 0.56 & 0.28 & 0.51 & 0.25 & 0.65 & 0.63 \\
Watertight index & 0.40 & 0.45 & 0.34 & 0.30 & 0.97 & 0.97 \\
Airtight index & 143 & 106 & 131 & 106 & 70 & 80 \\
Last group's time (sec.) & & & & & &
\end{tabular}

Table 3. Computational comparisons for the damaged-ship scenarios on the "USS Stark case."

\begin{tabular}{|c|c|c|c|c|c|c|}
\hline \multirow{3}{*}{$\begin{array}{l}\text { Ship } \\
\text { Watch condition, material } \\
\text { condition } \\
\text { Model }\end{array}$} & \multicolumn{4}{|c|}{ USS Stark } & \multirow{2}{*}{\multicolumn{2}{|c|}{$\begin{array}{c}\text { USS Cole } \\
\text { In-port, } \\
\text { XRAY }\end{array}$}} \\
\hline & \multicolumn{2}{|c|}{$\begin{array}{c}\text { General Quarters, } \\
\text { ZEBRA }\end{array}$} & \multicolumn{2}{|c|}{$\begin{array}{c}\text { Wartime Cruising, } \\
\text { YOKE }\end{array}$} & & \\
\hline & Static & Dynamic & Static & Dynamic & Static & Dynamic \\
\hline Objective value $\hat{z}$ & 0.40 & 0.33 & 0.49 & 0.36 & 0.60 & 0.48 \\
\hline Evacuation-time index $\hat{T}$ & 0.36 & 0.33 & 0.49 & 0.38 & 0.55 & 0.41 \\
\hline Ship-integrity index $\hat{I}$ & 0.52 & 0.33 & 0.49 & 0.31 & 0.74 & 0.72 \\
\hline Avg. evac. time (sec.) & 64 & 59 & 88 & 68 & 99 & 73 \\
\hline Watertight index & 0.56 & 0.29 & 0.54 & 0.29 & 0.66 & 0.63 \\
\hline Airtight index & 0.40 & 0.44 & 0.36 & 0.40 & 0.97 & 0.97 \\
\hline Last group’s time (sec.) & 143 & 158 & 397 & 270 & 507 & 180 \\
\hline
\end{tabular}


Table 4. Objective function, evacuation time and ship integrity for the new design for intact-ship scenarios: Comparison in “\% of improvement" with respect to DERS' results without the design change. The material condition is XRAY in all cases.

\begin{tabular}{l|rrr} 
Watch condition & $\begin{array}{c}\text { General } \\
\text { Quarters }\end{array}$ & $\begin{array}{c}\text { Wartime } \\
\text { Cruising }\end{array}$ & \multicolumn{1}{c}{ In-port } \\
\hline Objective value $\hat{z}$ & $4.8 \%$ & $26.1 \%$ & $38.7 \%$ \\
Avg. evac. time (sec.) & $1.7 \%$ & $27.1 \%$ & $32.1 \%$ \\
Last group's time (sec.) & $12.3 \%$ & $1.9 \%$ & $1.3 \%$ \\
Watertight index & $9.2 \%$ & $25.8 \%$ & $77.3 \%$ \\
Airtight index & $28.1 \%$ & $14.5 \%$ & $1.7 \%$
\end{tabular}

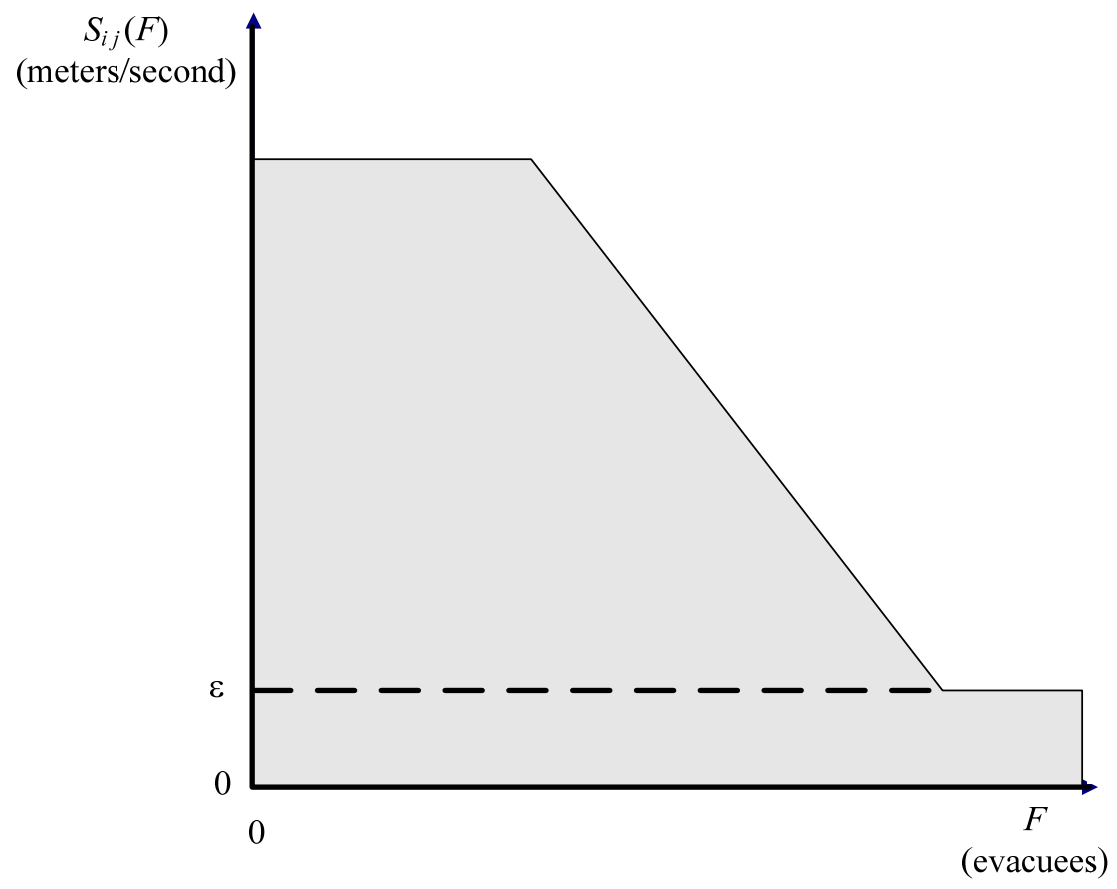

Figure 1. Representative movement-speed function $S_{i j}(F)$, where $F$ denotes the concurrent flow of crewmembers on arc $(i, j)$. A minimum speed $\varepsilon>0$ is used to avoid divisions by 0 . 

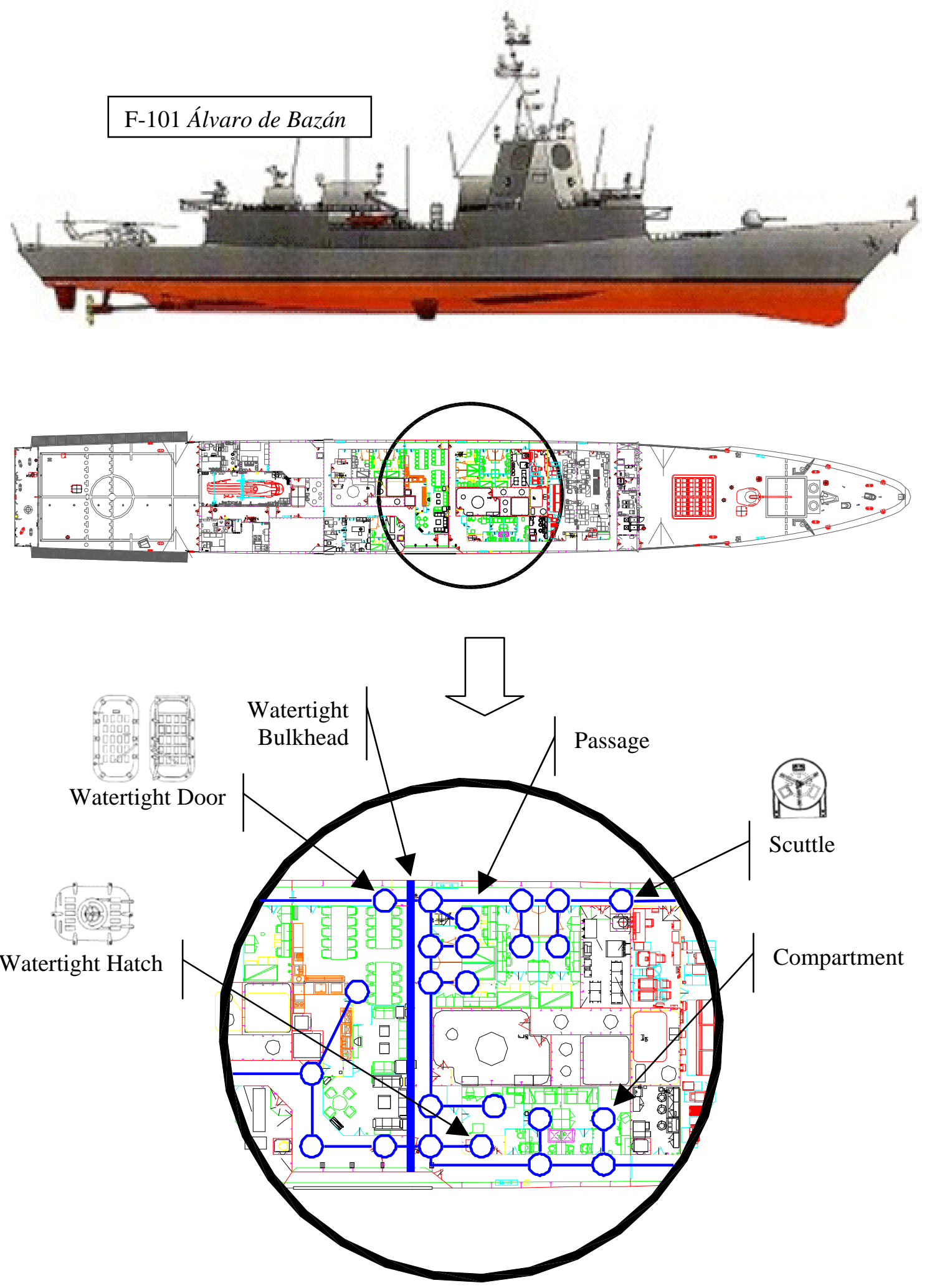

Figure 2. Sample DERS network components for the Álvaro de Bazán created from technical drawings. The Álvaro de Bazán has a length, beam and draft of 147, 18 and 10 meters, respectively, and a displacement of 5,800 tonnes. 
DRAFT, 1 July 2008

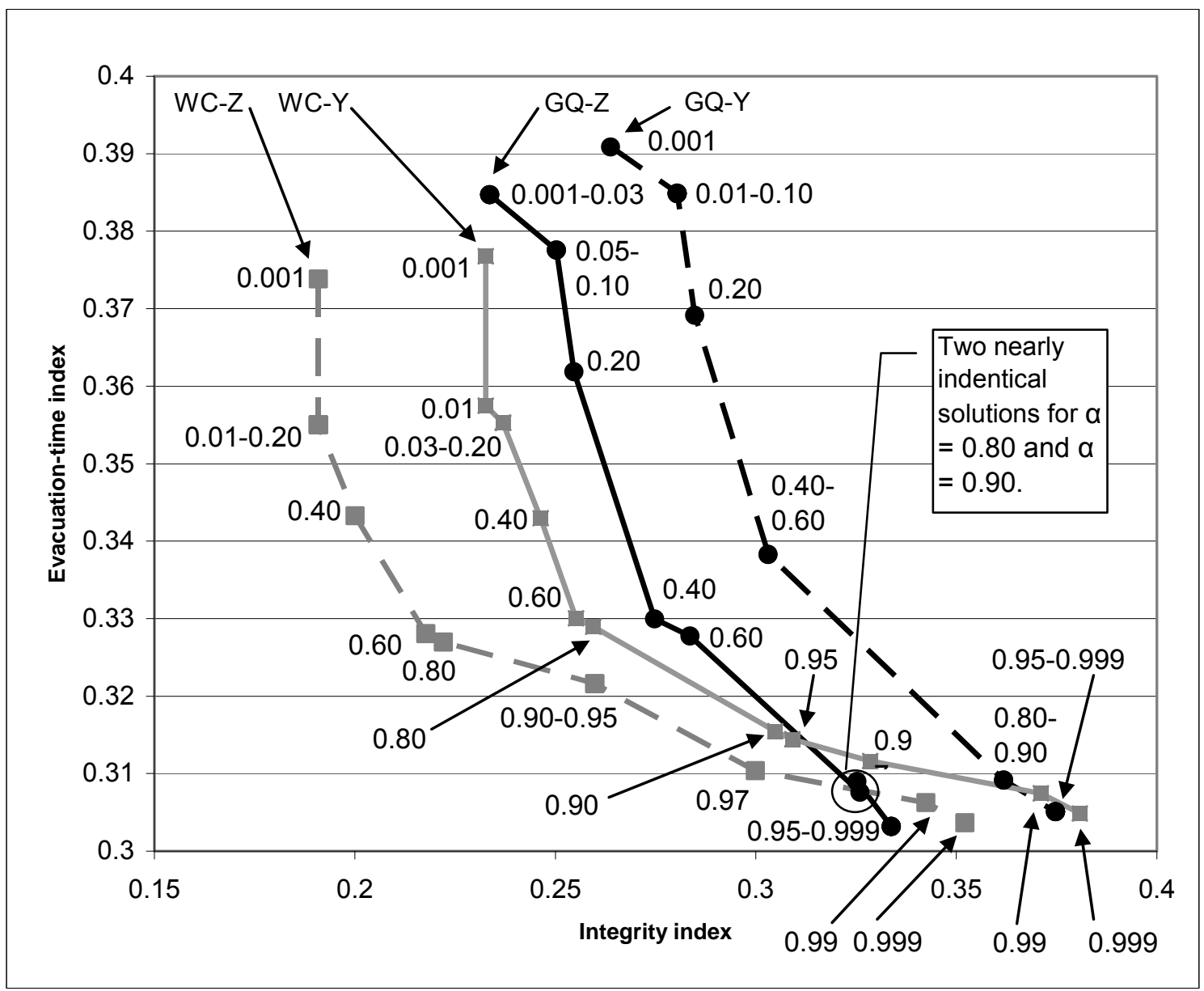

Figure 3. Approximate efficient frontiers for the evacuation-time index versus the ship-integrity index as computed by DERS for an intact ship under different watch and material conditions. The plots cover values of $\alpha \in(0.001,0.01,0.03,0.05,0.10,0.20,0.40,0.60,0.80,0.90,0.95,0.97,0.99,0.999)$ - these values are listed next to the points they correspond to-for each of four combinations of watch condition and material condition. The first two combinations, General Quarters with Zebra (GQ-Z) and Wartime Cruising with Yoke (WC-Y) are standard. The second two, General Quarters with Yoke (GQ-Y) and Wartime Cruising with Zebra (WC-Z), are artificial combinations that are used to help interpret results. 